ANNALES

POLONICI MATHEMATICI

$89.1(2006)$

\title{
On a functional equation with derivative and symmetrization
}

\author{
by ADAm Bobrowski (Lublin and Katowice) and \\ MąGORZATA KubalińsKa (Lublin)
}

\begin{abstract}
We study existence, uniqueness and form of solutions to the equation $\alpha g-\beta g^{\prime}+\gamma g_{\mathrm{e}}=f$ where $\alpha, \beta, \gamma$ and $f$ are given, and $g_{\mathrm{e}}$ stands for the even part of a searched-for differentiable function $g$. This equation emerged naturally as a result of the analysis of the distribution of a certain random process modelling a population genetics phenomenon.
\end{abstract}

1. Introduction. Let $\mathrm{BUC}(\mathbb{R})$ be the space of bounded, uniformly continuous functions on $\mathbb{R}$. In [2] we were led (see below for more details) to considering the following functional equation in this space:

$$
\alpha g-\beta g^{\prime}+\gamma g_{\mathrm{e}}=f,
$$

where $g \in \mathrm{BUC}(\mathbb{R})$ is a searched-for differentiable function, $g_{\mathrm{e}}$ is its even part $g_{\mathrm{e}}(t)=\frac{1}{2}(g(t)+g(-t))$, and the function $f \in \mathrm{BUC}(\mathbb{R})$ and the constants $\alpha, \beta>0$ and $\gamma>-\alpha$ are given. We showed that the unique solution to this equation is

$$
g=\frac{1}{\beta}\left(R_{\mu} f\right)^{\prime}+\frac{\alpha}{\beta^{2}} R_{\mu} f_{\mathrm{e}}+\frac{\mu}{\alpha} R_{\mu} f_{\mathrm{o}}=\frac{1}{\beta}\left(R_{\mu} f\right)^{\prime}+\frac{\alpha}{\beta^{2}} R_{\mu} f+\frac{\gamma}{\beta^{2}} R_{\mu} f_{\mathrm{o}},
$$

where $\mu=\alpha(\alpha+\gamma) / \beta^{2}, f_{\mathrm{o}}=f-f_{\mathrm{e}}$ is the odd part of $f$, and

$$
R_{\lambda} f(t)=\frac{1}{2 \sqrt{\lambda}} \int_{-\infty}^{\infty} e^{-\sqrt{\lambda}|s|} f(t+s) d s, \quad \lambda>0 .
$$

It is interesting that although (1) is a "first order" equation, its solutions are given in terms of $R_{\lambda}, \lambda>0$, which is the resolvent of the second order differential operator $f \mapsto f^{\prime \prime}$ (with natural domain). The reason is that (1)

2000 Mathematics Subject Classification: Primary 39B05; Secondary 60G35, 92D10, $47 \mathrm{D} 03$.

Key words and phrases: functional equation, differential equation, Cauchy problem, semigroups of operators, genetic drift. 
is equivalent to the system

$$
\left\{\begin{array}{l}
\alpha g_{\mathrm{e}}-\beta\left(g_{\mathrm{o}}\right)^{\prime}+\gamma g_{\mathrm{e}}=f_{\mathrm{e}} \\
\alpha g_{\mathrm{o}}-\beta\left(g_{\mathrm{e}}\right)^{\prime}=f_{\mathrm{o}}
\end{array}\right.
$$

of differential equations for the components $g_{\mathrm{e}}$ and $g_{\mathrm{o}}$ of $g$.

In this paper we show, by considering two generalizations of equation (1), that the result obtained in [2] is a particular case of a more general principle. In Section 2, we find the general solution of (1) for arbitrary real constants $\alpha, \beta$ and $\gamma$, in Section 3 we consider (1) with variable coefficients and in Section 4 we consider (1) in an abstract Banach space.

The origin of equation (1) is related to the work on mathematical description of genetic drift, a phenomenon known in population genetics. Genetic drift is often defined as a loss of variability of genetic material in a finite population, caused by random events, such as death of a member carrying this material. In the basic Fisher-Wright model of this phenomenon (see e.g. $[3,6,17])$, the population consists of a fixed number of individuals who live, each independently of the others, for a random, exponential time. At the death of an individual, an exact copy of a randomly chosen member of population replaces the member just deceased. In the absence of other genetic forces, this procedure leads to gradual loss of variability in the population. In the real populations and in more complicated models, the action of genetic drift is counteracted by other forces such as mutation, recombination and selection, with the drift striving to reduce the variability being constantly introduced by these forces. Viewed backwards, genetic drift is seen as a stochastic process of coalescence of ancestral lines in the time running backwards - this is the central idea of the epoch-making papers by Kingman [12] and Tajima [16]. To be more specific, the reproductive mechanism makes it clear that some random time ago there lived a common ancestor of the whole Fisher-Wright population and the variability existing today is the sole result of the action of other genetic forces (such as mutations) on the genetic material of his descendants; the descendants of the other members of the population existing at the time of the common ancestor die out in the meantime and this material is lost from the population.

In the case of a sample of two individuals, we deal with two stochastic processes (modelling mutations on the genealogical lines) that, conditional on their current states, evolve independently and with the same transition probabilities, and yet are dependent by the fact that some random time ago they evolved as a single process; their independent evolution started at the random time of split in the past. It is interesting that although in the case where these processes are Lévy processes there is no stationary distribution for the pair, the distribution of the relative difference stabilizes in time (see [11]). In [2] we show that the limit distribution of the process may 
be derived by means of the resolvent of a semigroup of operators related to the process of differences between two independent Lévy processes starting from the same initial state. As an example, we consider the telegraph process

$$
\mathrm{p}_{t}=\left(v \int_{0}^{t}(-1)^{M(s)} d s,(-1)^{M(t)}\right), \quad t \geq 0,
$$

where $a$ and $v$ are given positive constants and $\{M(t), t \geq 0\}$ is a Poisson process with $\mathbb{E} M(t)=$ at. (The reason why $\mathrm{p}_{t}, t \geq 0$, is called the telegraph process is that, as shown by M. Kac [10] inspired by S. Goldstein [8], the solutions to the telegraph equation may be expressed by means of the expected value of $\int_{0}^{t}(-1)^{M(s)} d s, t \geq 0$.) The telegraph process is a Lévy process when considered as a process with values in a non-commutative locally compact group $\mathbb{G}=\mathbb{R} \times\{-1,1\}$ with topology induced from $\mathbb{R}^{2}$ and multiplication rule $(\tau, k)(\xi, l)=(l \tau+\xi, k l)$ (see [14]). In [2] we show that the generator $A$ of the semigroup related to the process of differences is given by $A\left(f_{1}, f_{2}\right)=\left(2 a\left(f_{2}\right)_{\mathrm{e}}-2 a f_{1}, 2 v f_{2}^{\prime}+2 a\left(f_{1}\right)_{\mathrm{e}}-2 a f_{2}\right)$. The semigroup acts in $\mathrm{BUC}(\mathbb{R}) \times \mathrm{BUC}(\mathbb{R})$ (which is isometrically isomorphic to the space of bounded functions on $\mathbb{G}$ that are uniformly continuous with respect to the left and right uniform structures on $\mathbb{G}$ ), and the domain $\mathcal{D}$ of $A$ is the set of pairs $\left(f_{1}, f_{2}\right)$ where both $f_{i}$ belong to $\operatorname{BUC}(\mathbb{R})$, and $f_{2}$ is differentiable with $f_{2}^{\prime} \in \mathrm{BUC}(\mathbb{R})$. To find the resolvent of $A$ we needed to find, given $\lambda>0$ and $\left(f_{1}, f_{2}\right) \in \mathrm{BUC}(\mathbb{R}) \times \mathrm{BUC}(\mathbb{R})$, a pair $\left(g_{1}, g_{2}\right) \in \mathcal{D}$ such that

$$
\left\{\begin{array}{l}
\lambda g_{1}-2 a\left(g_{2}\right)_{\mathrm{e}}+2 g_{1}=f_{1}, \\
\lambda g_{2}-2 v g_{2}^{\prime}-2 a\left(g_{1}\right)_{\mathrm{e}}+2 a g_{2}=f_{2} .
\end{array}\right.
$$

Substituting $g_{1}=\frac{2 a}{\lambda+2 a}\left(g_{2}\right)_{\mathrm{e}}+\frac{1}{\lambda+2 a} f_{1}$ into the second equation, we obtain

$$
(\lambda+2 a) g_{2}-2 v g_{2}^{\prime}-\frac{4 a^{2}}{\lambda+2 a}\left(g_{2}\right)_{\mathrm{e}}=\frac{2 a}{\lambda+2 a}\left(f_{1}\right)_{\mathrm{e}}+f_{2} .
$$

This is equation (1) with $\alpha=\lambda+2 a, \beta=2 v, \gamma=-\frac{4 a^{2}}{\lambda+2 a}, g=g_{2}$ and $f=\frac{2 a}{\lambda+2 a}\left(f_{1}\right)_{\mathrm{e}}+f_{2}$.

2. The general solution to (1) with constant coefficients. Consider the differential equation

$$
\alpha g(t)-\beta g^{\prime}(t)+\gamma g_{\mathrm{e}}(t)=f(t) \quad \text { for all } t \in \mathbb{R},
$$

where $f$ is a given function, $g$ is a searched-for differentiable function, $g_{\mathrm{e}}$ is its even part: $g_{\mathrm{e}}(t)=\frac{1}{2}(g(t)+g(-t))$, and $\alpha, \beta$ and $\gamma$ are real numbers with $\alpha^{2}+\beta^{2}+\gamma^{2}>0$. 
Let $\widehat{g}(t)=g(-t), t \in \mathbb{R}$. Replacing $g(t)$ by $g(-t)$ in (6) we obtain

$$
\alpha \widehat{g}(t)+\beta \widehat{g}^{\prime}(t)+\gamma g_{\mathrm{e}}(t)=\widehat{f}(t), \quad t \in \mathbb{R} .
$$

Adding (6) and (7) and dividing by 2 gives

$$
\alpha g_{\mathrm{e}}(t)-\beta g_{\mathrm{o}}^{\prime}(t)+\gamma g_{\mathrm{e}}(t)=f_{\mathrm{e}}(t),
$$

where $g_{\mathrm{o}}$ is the odd part of $g: g_{\mathrm{o}}=g-g_{\mathrm{e}}$. Analogously, subtracting (7) from (6) and dividing by 2 yields

$$
\alpha g_{\mathrm{o}}(t)-\beta g_{\mathrm{e}}^{\prime}(t)=f_{\mathrm{o}}(t) .
$$

Conversely, (8) and (9) imply (6). Hence, (6) is equivalent to the following system of differential equations with constant coefficients:

$$
\left\{\begin{array}{l}
\alpha g_{\mathrm{e}}(t)-\beta g_{\mathrm{o}}^{\prime}(t)+\gamma g_{\mathrm{e}}(t)=f_{\mathrm{e}}(t), \\
\alpha g_{\mathrm{o}}(t)-\beta g_{\mathrm{e}}^{\prime}(t)=f_{\mathrm{o}}(t)
\end{array}\right.
$$

In order to find the general solution to this system we need to consider the following cases:

2.1. $\beta=0$. This case splits naturally into the following three subcases. (We note that $\alpha=0$ and $\alpha+\gamma=0$ is impossible, for this would imply that $\alpha=\beta=\gamma=0$.)

1. Suppose $\alpha \neq 0$ and $\alpha+\gamma \neq 0$. Then the system takes the form

$$
\left\{\begin{array}{l}
g_{\mathrm{e}}(t)=\frac{1}{\alpha+\gamma} f_{\mathrm{e}}(t), \\
g_{\mathrm{o}}(t)=\frac{1}{\alpha} f_{\mathrm{o}}(t),
\end{array}\right.
$$

and the solution of (6) is $g=g_{\mathrm{e}}+g_{\mathrm{o}}=\frac{1}{\alpha+\gamma} f_{\mathrm{e}}+\frac{1}{\alpha} f_{\mathrm{o}}$.

2. Suppose $\alpha=0$ and $\gamma \neq 0$. Then the system takes the form

$$
\left\{\begin{array}{l}
\gamma g_{\mathrm{e}}(t)=f_{\mathrm{e}}(t) \\
f_{\mathrm{o}}(t)=0
\end{array}\right.
$$

A solution to this system exists if and only if $f$ is even. In that case $g$ is given by $g=(1 / \gamma) f_{\mathrm{e}}+h=(1 / \gamma) f+h$, where $h$ is an arbitrary odd function.

3. Suppose $\alpha \neq 0$ and $\alpha+\gamma=0$. Then the system takes the form

$$
\left\{\begin{array}{l}
f_{\mathrm{e}}(t)=0, \\
g_{\mathrm{o}}(t)=\frac{1}{\alpha} f_{\mathrm{o}}(t) .
\end{array}\right.
$$

A solution to this system exists if and only if $f$ is odd. In that case $g$ is given by $g=(1 / \alpha) f_{\mathrm{o}}+h=(1 / \alpha) f+h$, where $h$ is an arbitrary even function. 
2.2. $\beta \neq 0$. In this case we may write

$$
\left\{\begin{array}{l}
g_{\mathrm{o}}^{\prime}(t)=\frac{\alpha+\gamma}{\beta} g_{\mathrm{e}}(t)-\frac{1}{\beta} f_{\mathrm{e}}(t), \\
g_{\mathrm{e}}^{\prime}(t)=\frac{\alpha}{\beta} g_{\mathrm{o}}(t)-\frac{1}{\beta} f_{\mathrm{o}}(t) .
\end{array}\right.
$$

Introducing

$$
A=\left(\begin{array}{cc}
0 & (\alpha+\gamma) / \beta \\
\alpha / \beta & 0
\end{array}\right)
$$

and using $A^{2 n}=\left(\alpha(\alpha+\gamma) / \beta^{2}\right)^{n} I, n \geq 1$, we find that

$$
e^{t A}=\cosh \left(t \frac{\sqrt{\alpha(\alpha+\gamma)}}{\beta}\right) I+\frac{\beta}{\sqrt{\alpha(\alpha+\gamma)}} \sinh \left(t \frac{\sqrt{\alpha(\alpha+\gamma)}}{\beta}\right) A,
$$

provided $\alpha(\alpha+\gamma) \neq 0$. For $\alpha(\alpha+\gamma)=0$, we have $e^{t A}=I+A t$. Hence, the general solution $\left(\begin{array}{l}g_{\mathrm{o}}(t) \\ g_{\mathrm{e}}(t)\end{array}\right)$ to $(10)$ is

$$
\begin{array}{r}
\left(\begin{array}{c}
C_{1} \cosh (t \sqrt{\alpha(\alpha+\gamma)} / \beta)+C_{2} \sqrt{(\alpha+\gamma) / \alpha} \sinh (t \sqrt{\alpha(\alpha+\gamma)} / \beta) \\
C_{1} \sqrt{\alpha /(\alpha+\gamma)} \sinh (t \sqrt{\alpha(\alpha+\gamma)} / \beta)+C_{2} \cosh (t \sqrt{\alpha(\alpha+\gamma)} / \beta)
\end{array}\right) \\
-\frac{1}{\beta}\left(\begin{array}{c}
\int_{0}^{t} f_{\mathrm{e}}(t-s) \cosh (s \sqrt{\alpha(\alpha+\gamma)} / \beta) d s \\
\sqrt{\alpha /(\alpha+\gamma)} \int_{0}^{t} f_{\mathrm{e}}(t-s) \sinh (s \sqrt{\alpha(\alpha+\gamma)} / \beta) d s
\end{array}\right) \\
-\frac{1}{\beta}\left(\begin{array}{c}
\sqrt{(\alpha+\gamma) / \alpha} \int_{0}^{t} f_{\mathrm{o}}(t-s) \sinh (s \sqrt{\alpha(\alpha+\gamma)} / \beta) d s \\
\int_{0}^{t} f_{\mathrm{o}}(t-s) \cosh (s \sqrt{\alpha(\alpha+\gamma)} / \beta) d s
\end{array}\right)
\end{array}
$$

and so $g$ is given by

$$
\begin{aligned}
g(t)= & \left(C_{1}+C_{2}\right) \cosh \left(t \frac{\sqrt{\alpha(\alpha+\gamma)}}{\beta}\right) \\
& +\frac{\left(C_{1}+C_{2}\right) \alpha+C_{2} \gamma}{\sqrt{\alpha(\alpha+\gamma)}} \sinh \left(t \frac{\sqrt{\alpha(\alpha+\gamma)}}{\beta}\right) \\
& -\frac{1}{\beta} \int_{0}^{t} f(t-s) \cosh \left(s \frac{\sqrt{\alpha(\alpha+\gamma)}}{\beta}\right) d s \\
& -\frac{\sqrt{\alpha(\alpha+\gamma)}}{\alpha \beta} \int_{0}^{t} f(t-s) \sinh \left(s \frac{\sqrt{\alpha(\alpha+\gamma)}}{\beta}\right) d s \\
& +\frac{\gamma}{\beta \sqrt{\alpha(\alpha+\gamma)}} \int_{0}^{t} f_{\mathrm{e}}(t-s) \sinh \left(s \frac{\sqrt{\alpha(\alpha+\gamma)}}{\beta}\right) d s .
\end{aligned}
$$


If $(\alpha+\gamma) \alpha=0$, then

$$
\begin{aligned}
g(t)= & \left(C_{1}+C_{2}\right)+\left(\frac{\alpha+\gamma}{\beta} C_{2}+\frac{\alpha}{\beta} C_{1}\right) t-\frac{1}{\beta} \int_{0}^{t} f(t-s) d s \\
& -\frac{\alpha+\gamma}{\beta^{2}} \int_{0}^{t} s f(t-s) d s+\frac{\gamma}{\beta^{2}} \int_{0}^{t} s f_{\mathrm{e}}(t-s) d s .
\end{aligned}
$$

ExAMPLE 1. Let $f$ be a given member of $\mathrm{BUC}(\mathbb{R})$ and let $\alpha, \beta>0$, $\gamma>-\alpha$. Our result implies that in $\mathrm{BUC}(\mathbb{R})$ there exists exactly one solution to (1), given by (2). To see this, rewrite (11) as follows:

$$
\begin{aligned}
g(t)= & \widetilde{C}_{1} e^{t \sqrt{\mu}}+\widetilde{C}_{2} e^{-t \sqrt{\mu}} \\
& -\frac{\beta \sqrt{\mu}+\alpha}{2 \alpha \beta} \int_{0}^{t} f(s) e^{(t-s) \sqrt{\mu}} d s+\frac{\gamma}{2 \beta^{2} \sqrt{\mu}} \int_{0}^{t} f_{\mathrm{e}}(s) e^{(t-s) \sqrt{\mu}} d s \\
& +\frac{\beta \sqrt{\mu}-\alpha}{2 \alpha \beta} \int_{0}^{t} f(s) e^{-(t-s) \sqrt{\mu}} d s-\frac{\gamma}{2 \beta^{2} \sqrt{\mu}} \int_{0}^{t} f_{\mathrm{e}}(s) e^{-(t-s) \sqrt{\mu}} d s
\end{aligned}
$$

where

$$
\widetilde{C}_{1}=\frac{C_{1}+C_{2}}{2}+\frac{\alpha C_{1}+(\alpha+\gamma) C_{2}}{2 \beta \sqrt{\mu}}, \quad \widetilde{C}_{2}=\frac{C_{1}+C_{2}}{2}-\frac{\alpha C_{1}+(\alpha+\gamma) C_{2}}{2 \beta \sqrt{\mu}},
$$

and $\mu=\alpha(\alpha+\gamma) / \beta^{2}$. As in [3, p. 244] we check that the only choice for $\widetilde{C}_{1}$ and $\widetilde{C}_{2}$ that leads to $g$ in $\operatorname{BUC}(\mathbb{R})$ is

$$
\begin{aligned}
& \widetilde{C}_{1}=\frac{\beta \sqrt{\mu}+\alpha}{2 \alpha \beta} \int_{0}^{\infty} f(s) e^{-s \sqrt{\mu}} d s-\frac{\gamma}{2 \beta^{2} \sqrt{\mu}} \int_{0}^{\infty} f_{\mathrm{e}}(s) e^{-s \sqrt{\mu}} d s \\
& \widetilde{C}_{2}=\frac{\beta \sqrt{\mu}-\alpha}{2 \alpha \beta} \int_{-\infty}^{0} f(s) e^{s \sqrt{\mu}} d s-\frac{\gamma}{2 \beta^{2} \sqrt{\mu}} \int_{-\infty}^{0} f_{\mathrm{e}}(s) e^{s \sqrt{\mu}} d s .
\end{aligned}
$$

For these constants equation (12) becomes

$$
\begin{aligned}
g(t)= & \frac{\alpha}{2 \beta^{2} \sqrt{\mu}} \int_{-\infty}^{\infty} f(s) e^{-|t-s| \sqrt{\mu}} d s+\frac{\gamma}{2 \beta^{2} \sqrt{\mu}} \int_{-\infty}^{\infty} f_{\mathrm{o}}(s) e^{-|t-s| \sqrt{\mu}} d s \\
& -\frac{1}{2 \beta} \int_{-\infty}^{t} f(s) e^{-(t-s) \sqrt{\mu}} d s+\frac{1}{2 \beta} \int_{t}^{\infty} f(s) e^{(t-s) \sqrt{\mu}} d s .
\end{aligned}
$$

Since $\left(R_{\mu} f\right)^{\prime}(t)=-\frac{1}{2} \int_{-\infty}^{t} f(s) e^{-(t-s) \sqrt{\mu}} d s+\frac{1}{2} \int_{t}^{\infty} f(s) e^{(t-s) \sqrt{\mu}} d s$, this agrees with (2). 
3. Equation (1) with variable coefficients. Let $a, b, c: \mathbb{R} \rightarrow \mathbb{R}$ be bounded, continuous functions. Consider the equation

$$
g^{\prime}=a g+b g_{\mathrm{e}}+c .
$$

Given $\omega>0$ we search for solutions of this problem in the class $\mathbb{B}_{\omega}$ of continuous functions $g$ such that $\sup _{t \in \mathbb{R}} e^{-\omega|t|}|g(t)|$ is finite. We note that $\mathbb{B}_{\omega}$ is a Banach space with the norm

$$
\|g\|_{\omega}=\sup _{t \in \mathbb{R}} e^{-\omega|t|}|g(t)| \text {. }
$$

We refer to $\mathbb{B}_{\omega}$ as the Bielecki space (see $\left.[1,4]\right)$.

Proposition 1. Fix $\omega>2 M$ where

$$
M=\max \left\{\sup _{t \in \mathbb{R}}|a(t)|, \sup _{t \in \mathbb{R}}|b(t)|, \sup _{t \in \mathbb{R}}|c(t)|\right\} .
$$

The Cauchy problem for the equation (14) with initial condition $g(0)=\kappa \in \mathbb{R}$ has a unique solution in the Bielecki space $\mathbb{B}_{\omega}$.

Proof. A function $g$ satisfies (14) with initial condition $g(0)=\kappa$ iff it is a fixed point of the operator $F$ in $\mathbb{B}_{\omega}$ given by

$$
[F(g)](t)=\kappa+\int_{0}^{t} a(s) g(s) d s+\int_{0}^{t} b(s) g_{\mathrm{e}}(s) d s+\int_{0}^{t} c(s) d s, \quad t \in \mathbb{R} .
$$

We note that $F$ indeed maps $\mathbb{B}_{\omega}$ into itself. For, clearly, $F(g)$ is a continuous function. Moreover, $\left|\kappa+\int_{0}^{t} c(s) d s\right| \leq|\kappa|+M|t|$, so that $t \mapsto \kappa+\int_{0}^{t} c(s) d s$ is a member of $\mathbb{B}_{\omega}, \omega>0$. Also, for any $t \in \mathbb{R}$ and $g \in \mathbb{B}_{\omega}$,

$$
\begin{aligned}
\left|e^{-\omega|t|} \int_{0}^{t} b(s) g_{\mathrm{e}}(s) d s\right| & \leq M\left|\int_{0}^{t} e^{-\omega(|t|-|s|)} e^{-\omega|s|}\right| g_{\mathrm{e}}(s)|d y| \\
& \leq M\left\|g_{\mathrm{e}}\right\|_{\omega}\left|\int_{0}^{t} e^{-\omega(|t|-|s|)} d s\right| \leq M \omega^{-1}\|g\|_{\omega},
\end{aligned}
$$

which shows that the third term in (15) is a member of $\mathbb{B}_{\omega}$. Similarly, we show that so is the second term there.

A calculation similar to the one in (16) shows that $\|F(f)-F(g)\|_{\omega}<$ $(2 M / \omega)\|f-g\|_{\omega}$. Hence, for $\omega>2 M, F$ is a contraction in $\mathbb{B}_{\omega}$ and so, by the Banach fixed point theorem, there exists a unique fixed point of $F$.

Remark 1. Since $\mathbb{B}_{\omega} \subset \mathbb{B}_{\omega^{\prime}}$ for $\omega<\omega^{\prime}$, by Proposition 1 , there can be no more than one solution to $(14)$ in $\mathbb{B}_{\omega}$ where $\omega \leq 2 M$. Moreover, if $g$ is a fixed point of $F$ in $\mathbb{B}_{\omega}$ for some $\omega$ then it is a fixed point of $F$ in all $\mathbb{B}_{\omega^{\prime}}$ where $\omega^{\prime}>\omega$. Hence our proposition states that there exists a unique solution to the Cauchy problem related to (14) and this solution belongs to $\mathbb{B}_{\omega}$ for all $\omega>2 M$. 
Although it seems that a closed form of the general solution to (14) is not available, we can still show that this equation is equivalent to a system of two ordinary differential equations for the even and odd parts of $g$. To this end we note first that, as can be checked directly, for any functions $f$ and $g$ on $\mathbb{R},(f g)_{\mathrm{e}}=f g_{\mathrm{e}}-\widehat{g} f_{\mathrm{o}}$ and $(f g)_{\mathrm{o}}=f g_{\mathrm{e}}-\widehat{g} f_{\mathrm{e}}$. Hence, calculating the even part of both sides of equation (14) and noting that $\left(g^{\prime}\right)_{\mathrm{e}}=g_{\mathrm{o}}^{\prime}$, we obtain

$$
\begin{aligned}
g_{\mathrm{o}}^{\prime} & =g a_{\mathrm{e}}-\widehat{a} g_{\mathrm{o}}+g_{\mathrm{e}} b_{\mathrm{e}}+c_{\mathrm{e}}=g_{\mathrm{e}} a_{\mathrm{e}}+g_{\mathrm{o}} a_{\mathrm{e}}-\widehat{a} g_{\mathrm{o}}+g_{\mathrm{e}} b_{\mathrm{e}}+c_{\mathrm{e}} \\
& =g_{\mathrm{e}}[a+b]_{\mathrm{e}}+g_{\mathrm{o}} a_{\mathrm{o}}+c_{\mathrm{e}} .
\end{aligned}
$$

Analogously, $g_{\mathrm{e}}^{\prime}=g_{\mathrm{e}}[a+b]_{\mathrm{o}}+a_{\mathrm{e}} g_{\mathrm{o}}+c_{\mathrm{o}}$. Conversely, the equations for $g_{\mathrm{e}}$ and $g_{\mathrm{o}}$ determine the equation for $g$. In other words, (14) is equivalent to the following system of differential equations:

$$
\left(\begin{array}{c}
g_{\mathrm{o}} \\
g_{\mathrm{e}}
\end{array}\right)^{\prime}=\left(\begin{array}{ll}
a_{\mathrm{o}} & (a+b)_{\mathrm{e}} \\
a_{\mathrm{e}} & (a+b)_{\mathrm{o}}
\end{array}\right)\left(\begin{array}{l}
g_{\mathrm{o}} \\
g_{\mathrm{e}}
\end{array}\right)+\left(\begin{array}{c}
c_{\mathrm{e}} \\
c_{\mathrm{o}}
\end{array}\right) .
$$

Clearly, if $a$ and $b$ are constants equal to $\alpha / \beta$ and $\gamma / \beta$, respectively, this system reduces to $(2)$ with $c=-(1 / \beta) f$. Formula (17) may be used as a starting point for investigation of (14) in a more general case, where, for example, $a, b$ and $c$ are continuous but unbounded.

4. Equation (1) in an abstract Banach space. Suppose that $\mathbb{X}$ is a Banach space and $G$ is the generator (see e.g. [9]) of a strongly continuous group $\{U(t), t \in \mathbb{R}\}$ of equibounded operators in $\mathbb{X}$ :

$$
\sup _{t \in \mathbb{R}}\|U(t)\|=: M<\infty .
$$

Recall that the operators $C(t)=\frac{1}{2}(U(t)+U(-t)), t \in \mathbb{R}$, form a strongly continuous cosine operator function (see e.g. $[7,15])$ so that the cosine functional equation $2 C(t) C(s)=C(t+s)+C(t-s), s, t \in \mathbb{R}$, is satisfied. The generator of $\{C(t), t \in \mathbb{R}\}$, defined as $\lim _{h \rightarrow 0} \frac{2}{h^{2}}[C(h) f-f]$ for all $f \in \mathbb{X}$ for which this limit exists, can be checked to be equal to $G^{2}$ with natural domain (see e.g. [7, p. 77], cf. [13]). By the Sova generation theorem ([7, 15]), all positive $\lambda$ belong to the resolvent set of $G^{2}$ and

$$
\begin{aligned}
\lambda\left(\lambda^{2}-G^{2}\right)^{-1} & =\int_{0}^{\infty} e^{-\lambda t} C(t) d t \\
& =\frac{1}{2} \int_{0}^{\infty} e^{-\lambda t} U(t) d t+\frac{1}{2} \int_{0}^{\infty} e^{-\lambda t} U(-t) d t \\
& =\frac{1}{2}(\lambda-G)^{-1}+\frac{1}{2}(\lambda+G)^{-1}
\end{aligned}
$$

(all integrals in the strong topology). 
Suppose also that $\mathbb{X}$ is a direct sum of two subspaces: $\mathbb{X}_{P}$ and $\mathbb{X}_{Q}$ where the projections $P\left(\right.$ on $\mathbb{X}_{P}$ ) and $Q$ (on $\mathbb{X}_{Q}$ ) are bounded in norm by 1 . By the Phillips perturbation theorem [9], for any $a \in \mathbb{R}$, both $G_{a}:=G+a P$ and $-G_{a}$ generate strongly continuous semigroups, say $\left\{S_{a}(t), t \geq 0\right\}$ and $\left\{S_{a}^{-}(t), t \geq 0\right\}$, respectively, such that $\left\|S_{a}(t)\right\|,\left\|S_{a}^{-}(t)\right\| \leq M e^{|a| t}, t \geq 0$. Hence, by the Hille-Yosida generation theorem for groups $([5,9]), G_{a}$ is the generator of a strongly continuous group $\left\{S_{a}(t), t \in \mathbb{R}\right\}$ of operators in $\mathbb{X}$ and $\left\|S_{a}(t)\right\| \leq M e^{|a t|}, t \in \mathbb{R}$. In particular, any $\lambda \in \mathbb{R}$ such that $|\lambda|>|a|$ belongs to the resolvent set of $G_{a}$.

Suppose finally that for $f$ in the domain $\mathcal{D}(G)$ of $G, P f$ belongs to $\mathcal{D}(G)$ and $G P f=Q G f$. This implies that $Q f$ belongs to $\mathcal{D}(G)$ and

$$
G Q f=G(f-P f)=G f-Q G f=P G f .
$$

Proposition 2. Under the above assumptions, for $f \in \mathbb{X}$ and $|\lambda|>|a|$, the resolvent $R_{\lambda, a}:=\left(\lambda-G_{a}\right)^{-1}$ of $G_{a}$ is given by

$$
\begin{aligned}
R_{\lambda, a} f & =G\left(\mu-G^{2}\right)^{-1} f+\left(\mu-G^{2}\right)^{-1}\left(\lambda^{-1} \mu Q f+\lambda P f\right) \\
& =G\left(\mu-G^{2}\right)^{-1} f+\left(\mu-G^{2}\right)^{-1}(\lambda f-a Q f),
\end{aligned}
$$

where $\mu=\lambda(\lambda-a)>0$.

Proof. In view of the above remarks, for any $f \in \mathbb{X}$, the resolvent equation of $G_{a}$,

$$
\lambda g-G_{a} g=f,
$$

has a unique solution. We are to check that $g=R_{\lambda, a} f$ defined in (20) solves this equation. Clearly, $g \in \mathcal{D}(G)$. Moreover, a direct calculation shows that for any $h \in \mathbb{X}, k=P\left(\mu-G^{2}\right)^{-1} h$ solves $\mu k-G^{2} k=P h$. Since the unique solution to the last equation is $k=\left(\mu-G^{2}\right)^{-1} P h$, we see that $P$ commutes with $\left(\mu-G^{2}\right)^{-1}$. Hence, so does $Q$. Using

$$
G^{2}\left(\mu-G^{2}\right)^{-1} f=\mu\left(\mu-G^{2}\right)^{-1} f-f
$$

we obtain

$$
\begin{aligned}
G_{a} g=G g+a P g= & G^{2}\left(\mu-G^{2}\right)^{-1} f+G\left(\mu-G^{2}\right)^{-1}[\lambda f-a Q f+a Q f] \\
& +a \lambda\left(\mu-G^{2}\right)^{-1} P f \\
= & \left(\mu-G^{2}\right)^{-1}[\mu f+a \lambda P f]+\lambda G\left(\mu-G^{2}\right)^{-1} f-f .
\end{aligned}
$$

Since $\lambda g=\lambda G\left(\mu-G^{2}\right)^{-1} f+\left(\mu-G^{2}\right)^{-1}\left[\mu Q f+\lambda^{2} P f\right]$, the left-hand side of (21) with $g=R_{\lambda, a} f$ equals

$$
\left[-\mu+\lambda^{2}-a \lambda\right]\left(\mu-G^{2}\right)^{-1} P f+f=f,
$$

as desired. 
REMARK 2. Clearly, the way to derive relation (20) is to note that applying $P$ and $Q$ to both sides of (21) we obtain the following system of two equations that is equivalent to (21):

$$
\left\{\begin{array}{l}
\lambda P g-G Q g-a P g=P f \\
\lambda Q g-G P g=Q f
\end{array}\right.
$$

This system, under the additional assumption that $f \in \mathcal{D}(G)$, can be solved by plugging $Q g=\lambda^{-1}[Q f+G P g]$ into the first equation, and this leads to $(20)$.

EXAmple 2. Let $\mathbb{X}=\operatorname{BUC}(\mathbb{R})$ be the space of uniformly continuous functions on $\mathbb{R}$ with the supremum norm, and $\{U(t), t \in \mathbb{R}\}$ be the group of translations $U(t) f(s)=f(s+t), s, t \in \mathbb{R}$. Clearly, $\|U(t)\|=1, t \in \mathbb{R}$. The infinitesimal generator $G$ of this group is $G=d / d s$ with natural (maximal) domain.

Let $P$ be the projection of $\mathbb{X}$ onto the subspace $\mathbb{X}_{P}$ of even functions in $\mathbb{X}$, and $Q$ be the projection on the space of odd functions in $\mathbb{X}$. Obviously, $P f=f_{\mathrm{e}}$ and $Q f=f_{\mathrm{o}}$ and $\|P\|=\|Q\|=1$. Note that $P$ leaves the set of differentiable functions invariant and we have $G P f=Q G f$ for all differentiable functions $f$. In other words, the assumptions of Proposition 2 are satisfied. Therefore, the solution to equation (1) is given by $g=(1 / \beta) R_{\lambda, a} f$ with $a=-\gamma / \beta$ and $\lambda=\alpha / \beta$. To be more specific,

$$
g=\frac{1}{\beta}\left(R_{\mu}\right)^{\prime}+\frac{\alpha}{\beta^{2}} R_{\mu} f+\frac{\gamma}{\beta^{2}} R_{\mu} f_{\mathrm{o}}
$$

where

$$
\mu=\lambda(\lambda-a)=\alpha(\alpha+\gamma) / \beta^{2} \quad \text { and } \quad R_{\mu}=\left(\mu-G^{2}\right)^{-1} .
$$

Since

$$
\begin{aligned}
& (\lambda-G)^{-1} f(t)=\int_{0}^{\infty} e^{-\lambda s} f(t+s) d s, \\
& (\lambda+G)^{-1} f(t)=\int_{-\infty}^{0} e^{\lambda s} f(s+t) d t,
\end{aligned}
$$

relation (19) gives

$$
R_{\mu} f(t)=\frac{1}{2 \sqrt{\mu}} \int_{-\infty}^{\infty} e^{-\sqrt{\mu}|t|} f(t+s) d s .
$$

This establishes (2) once again.

REMARK 3. For $\gamma=0$, (1) reduces to an ordinary linear differential equation of first order with constant coefficients. Hence, in that case, (2) should reduce to the resolvent of a first-order differential operator. More 
generally, for $a=0,(20)$ should reduce to the resolvent of $G$. To check this we note that, by (19), $G(\lambda-G)^{-1} f=\lambda(\lambda-G)^{-1} f-f$ and $G(\lambda+G)^{-1} f=$ $f-\lambda(\lambda+G)^{-1} f$, and relation (20) may be written in the form

$$
R_{\lambda, a} f=\frac{1}{2}\left[(\sqrt{\mu}-G)^{-1} f-(\sqrt{\mu}+G)^{-1} f\right]+\left(\mu-G^{2}\right)^{-1}\left(\lambda^{-1} \mu Q f+\lambda P f\right) .
$$

For $a=0$ this reduces to

$$
\begin{aligned}
& \frac{1}{2}\left[(\lambda-G)^{-1} f-(\lambda+G)^{-1} f\right]+\left(\lambda^{2}-G^{2}\right)^{-1}(\lambda Q f+\lambda P f) \\
& \quad=\frac{1}{2}\left[(\lambda-G)^{-1} f-(\lambda+G)^{-1} f\right]+\lambda\left(\lambda^{2}-G^{2}\right)^{-1} f=(\lambda-G)^{-1} f
\end{aligned}
$$

(where we used (19) again), as expected.

Acknowledgements. This work was supported by the Polish Government research fund for 2005-2008, grant no. 1 P03A 04429 (0356/P03/ $2005 / 29)$.

\section{References}

[1] A. Bielecki, Une remarque sur la méthode de Banach-Caccioppoli-Tikhonov, Bull. Acad. Polon. Sci. 4 (1956), 261-268.

[2] A. Bobrowski, A Feller evolution family of operators that describes dynamics of characteristics of two individuals sampled from a Fisher-Wright population with varying size, in preparation.

[3] -, Functional Analysis for Probability and Stochastic Processes, Cambridge, 2005.

[4] R. E. Edwards, Functional Analysis. Theory and Applications, Holt, Rinehart and Winston, 1965, republished by Dover Publ., 1995.

[5] K.-J. Engel and R. Nagel, One-Parameter Semigroups for Linear Evolution Equations, Springer, 2000.

[6] W. Ewens, Mathematical Population Genetics. I. Theoretical Introduction, 2nd ed., Springer, 2004.

[7] H. O. Fattorini, Second Order Linear Differential Equations in Banach Spaces, North-Holland Math. Stud. 108, Elsevier, 1985.

[8] S. Goldstein, On diffusion of discontinuous movements, and on the telegraph equation, Quart. J. Mech. Appl. Math. 4 (1951), 129-156.

[9] E. Hille and R. S. Phillips, Functional Analysis and Semigroups, rev. ed., Amer. Math. Soc. Collloq. Publ. 31, 1957.

[10] M. Kac, Some Stochastic Problems in Physics and Mathematics, Magnolia Petroleum Co. Colloq. Lect. 2, 1956.

[11] J. F. C. Kingman, Coherent random walks arising in some genetic models, Proc. Roy. Soc. London Sect. A 351 (1976), 19-31.

[12] - The coalescent, Stochastic Process. Appl. 13 (1982), 235-248.

[13] J. Kisyński, On operator-valued solutions of d'Alembert's functional equation, I, Colloq. Math. 23 (1971), 107-114.

[14] - , On M. Kac's probabilistic formula for the solution of the telegraphist's equation, Ann. Polon. Math. 29 (1974), 259-272.

[15] M. Sova, Cosine operator functions, Dissertationes Math. 49 (1966). 
[16] F. Tajima, Evolutionary relationship of DNA sequences in finite populations, Genetics 105 (1983), 437-460.

[17] S. Tavaré and O. Zeitouni, Lectures on Probability Theory and Statistics (École d'Été de Probabilités de Saint-Flour XXXI, 2001), Lecture Notes in Math. 1837, Springer, 2004.

Institute of Mathematics

Polish Academy of Sciences

Katowice branch

Bankowa 14

40-007 Katowice, Poland

on leave from

Department of Mathematics

Faculty of Electrical Engineering

and Computer Science

Lublin University of Technology

Nadbystrzycka 38A

20-618 Lublin, Poland

E-mail: a.bobrowski@pollub.pl
Department of Computer Sciences

Faculty of Management and Fundamentals of Technology Lublin University of Technology

Nadbystrzycka 38 20-618 Lublin, Poland E-mail: m.kubalinska@pollub.pl

Received 13.10.2005

and in final form 19.2.2006 